\title{
DESENVOLVIMENTO DE CONSTRUTOS PARA OPERACIONALIZAÇÃO DO FRAMEWORK DOS 4IS
}

\author{
DEVELOPMENT OF CONSTRUCTS FOR AIS FRAMEWORK \\ OPERATIONALIZATION
}

Recebido em: 09 nov. 2018

Aprovado em: 09 out. 2019

Versão do autor aceita publicada online: 09 out. 2019

Publicado online: 12 maio 2021

Como citar esse artigo - American Psychological Association (APA):

Matos, L. dos S., \& Ensslin, S. R. (2021, jul./set.). Desenvolvimento de construtos para operacionalização do framework dos 4IS. Exacta, 19(3), 564-586.

https://doi.org/10.5585/exactaep.2021.10906

Submeta seu artigo para este periódico $\beta$

Dados Crossmark 


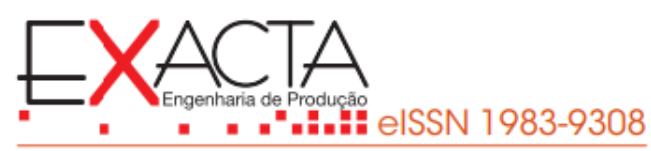

\title{
DESENVOLVIMENTO DE CONSTRUTOS PARA OPERACIONALIZAÇÃO DO FRAMEWORK DOS 4IS
}

\author{
DEVELOPMENT OF CONSTRUCTS FOR 4IS FRAMEWORK OPERATIONALIZATION
}

\section{Lucas dos Santos Matos $^{1}$}

\author{
${ }^{1}$ Doutor em Engenharia de Produção pela \\ Universidade Federal de Santa Catarina \\ Universidade Federal de Santa Catarina - \\ UFSC. \\ Florianópolis, SC - Brasil. \\ I.matos@ufsc.br \\ ${ }^{2}$ Doutora em Engenharia de Produção pela \\ Universidade Federal de Santa Catarina \\ Universidade Federal de Santa Catarina - \\ UFSC. \\ Florianópolis, SC - Brasil. \\ sensslin@gmail.com
}

Recebido em: 09 nov. 2018

Aprovado em: 09 out. 2019
Resumo: Esta pesquisa teve por objetivo desenvolver construtos para operacionalização dos processos de aprendizagem organizacional e permitir sua avaliação, com base no framework dos 4Is, de Crossan, Lane e White (1999). Para execução da pesquisa, realizou-se uma seleção sistematizada da literatura, identificando-se os artigos que operacionalizaram o framework, fazendo uso das bases internacionais Scopus e Web of Science. Com o apoio de um software gerenciador bibliográfico, EndNote, foram acessados, um a um, integralmente, os textos dos artigos que citaram o artigo base do framework dos 4ls. Desta forma, após análise foram identificados 27 artigos para compor o portfólio bibliográfico do estudo. A partir dos artigos do portfólio foram identificados os métodos e os procedimentos executados na pesquisa, permitindo o desenvolvimento de construtos consolidados a partir do conjunto de artigos que operacionalizaram o framework, descrevendo métodos e formas para avaliar os processos de aprendizagem organizacional - intuir, interpretar, integrar e institucionalizar.

Palavras-chave: Aprendizagem Organizacional. Framework dos 4Is. Construtos.

Abstract: This research aimed to develop constructs for the operationalization of organizational learning processes and to allow their evaluation, based on the 4Is framework of Crossan, Lane and White (1999). To perform the research, a systematized selection of the literature was carried out, identifying the articles that operationalized the framework, making use of the bases Scopus and Web of Science. With the support of a bibliographic management software, EndNote, the texts of the articles that quoted the basic article of the $4 \mathrm{Is}$ framework were accessed one by one in their entirety. In this way, after analysis, 27 articles were identified to compose the bibliographic portfolio for the study. From the portfolio articles, the methods and procedures performed in the researches were identified, allowing the development of consolidated constructs from the set of articles that operationalized the framework, describing methods and ways to evaluate the organizational learning processes - intuiting, interpreting, integrating and institutionalizing.

Keywords: Organizational learning. 4Is framework. Constructs. 
1 Introdução

Os processos de gestão nas organizações têm recebido atenção crescente devido à necessidade e demanda por informações precisas e tempestivas para o desenvolvimento dos negócios. Dentre esses processos, os estudos acerca da aprendizagem organizacional se apresentam como uma preocupação por parte da literatura sobre organizações (Bontis, Crossan \& Hulland, 2002; Brettel \& Rottenberger, 2013; Dutta \& Crossan, 2005; Dutton, Turner \& Lee-Kelley, 2014; Easterby-Smith, Crossan \& Nicolini, 2000; Jones \& Macpherson, 2006; Kostopoulos, Spanos \& Prastacos, 2013).

A Aprendizagem Organizacional (AO), por sua vez, lida com o desenvolvimento do conhecimento nas pessoas e nas organizações, relacionado à como o conhecimento é construído e disseminado na estrutura de uma empresa, refletindo em mudanças cognitivas e comportamentais nos envolvidos (Argote, 2011; Bontis, Crossan \& Hulland, 2002; Easterby-Smith, Crossan \& Nicolini, 2000; Dutta \& Crossan, 2005; Guzman, 2015; Lawrence et al., 2005; Zietsma et al., 2002). Trata-se de um processo dinâmico de desenvolvimento e disseminação do conhecimento, o qual, segundo Crossan, Lane e White (1999), pode ser concebido como um dos principais meios para promoção da renovação estratégica, a partir da assimilação de novos aprendizados e uso do que foi aprendido (March, 1991).

Salienta-se, porém, que os estudos relacionados à $\mathrm{AO}$ ainda buscam por uma convergência e um entendimento comum sobre o que vem a ser a aprendizagem organizacional. Estudos multidisciplinares relacionados ao tema e discussões segundo diferentes pontos de vista têm levado a um conhecimento disperso e divergente, prejudicando a sua consolidação na literatura (Argote, 2011; Easterby-Smith, Crossan \& Nicolini, 2000).

Neste sentido, com o objetivo de construir um fundamento sólido e consistente, Crossan, Lane e White (1999) desenvolveram seu estudo com a intenção de propor uma teoria sobre a AO. No entanto, a proposta inicial do desenvolvimento da teoria para o tema concluiu na proposição de um framework, composto pelos processos pelos quais se desenvolve a aprendizagem organizacional. Conforme o framework dos $4 \mathrm{Is}$, a aprendizagem na organização é vislumbrada segundo uma perspectiva evolutiva, como um processo dinâmico que envolve os níveis de aprendizagem individual, de grupo e organizacional (Crossan, Lane \& White, 1999). Dada a relevância do estudo (Berson, Da'as \& Waldman, 2015; Brettel \& Rottenberger, 2013; Jones \& Macpherson, 2006), tornou-se o artigo mais citado da Academy of Management Review (AMR) do período compreendido entre 2000 e 2009, o que levou ao convite, por parte da AMR, para realização de uma revisão, buscando refletir sobre os avanços relacionados ao campo e se uma teoria sobre AO teria surgido após a publicação do estudo (Crossan, Maurer \& White, 2011).

Segundo Crossan, Maurer e White (2011), ainda que diversos estudos tenham contribuído para o tema e para o framework, restam lacunas não preenchidas. Além da inexistência de uma teoria da $\mathrm{AO}$, 
pouco se discutiu sobre os processos de aprendizagem e como se operacionalizam cada um deles dentro das organizações. Assim, esse artigo busca contribuir para a literatura sobre AO com uma revisão dos estudos organizacionais que fizeram uso do framework dos 4Is (Crossan, Lane \& White, 1999), com o objetivo de desenvolver construtos para operacionalização dos processos de aprendizagem organizacional e permitir sua avaliação.

Dentre os métodos adotados para realização da pesquisa, procedeu-se a uma seleção sistematizada da literatura, para identificação de estudos e compor um portfólio composto pelos artigos que fizeram uso do framework dos $4 \mathrm{Is}$, e também se realizou a análise de conteúdo dos artigos do portfólio, buscando evidenciar os procedimentos para coleta dos dados relacionados aos processos de aprendizagem organizacional e métodos utilizados para avaliar a ocorrência desses processos. Após, a partir dos métodos identificados nos diferentes estudos, apresentam-se os construtos desenvolvidos para cada processo.

Espera-se que o estudo contribua para o tema relacionado à aprendizagem organizacional, fornecendo um arcabouço teórico, construído a partir do empirismo encontrado na literatura, e construtos para operacionalização e avaliação da aprendizagem organizacional. Desta forma, busca-se preencher aspectos pouco explorados sobre o framework dos $4 \mathrm{ls}$.

Quanto à estrutura deste trabalho, quatro outras seções são apresentadas. A segunda seção promove a fundamentação teórica do trabalho, relacionado à aprendizagem organizacional, principalmente no que diz respeito ao framework desenvolvido por Crossan, Lane e White (1999). A terceira seção apresenta os procedimentos metodológicos da pesquisa. Na seção quatro são explorados os resultados da pesquisa, apresentando os métodos identificados para coleta de dados nos estudos e a os construtos desenvolvidos para os processos de AO. As considerações finais são expostas na seção número cinco. Por fim, apresentam-se as referências bibliográficas.

\section{Fundamentação teórica}

O estímulo à melhoria contínua nas organizações, em resposta à evolução das demandas da sociedade e do mercado, tem provocado aumento na importância em se desenvolver processos de gestão mais assertivos, desenvolver conhecimento nas pessoas e, consequentemente, nas próprias organizações (Guzman, 2015; Kelliher, 2006; Lawrence et al., 2005). Essa evolução do conhecimento está intimamente conectada ao fenômeno de aprendizagem organizacional (Argote, 2011).

Para explicar o processo da aprendizagem organizacional, Crossan, Lane e White (1999) desenvolveram um framework conceitual, o qual busca evidenciar como o conhecimento flui em uma estrutura organizacional, perpassando pelos indivíduos, grupos e concluindo na institucionalização de informações para toda a organização. 
Visualiza-se, para fins deste trabalho, a aprendizagem organizacional como um processo dinâmico que ocorre para o desenvolvimento de conhecimento sobre aspectos e fatores inerentes ao ambiente e sobre a organização, promovendo o fenômeno da renovação estratégica nas organizações e conectando cognição com a efetividade das ações. Assim, considera-se que o que é aprendido deve ser materializado em ações (Crossan, Lane \& White, 1999; Crossan \& Berdrow, 2003), disseminando o conhecimento por toda a estrutura organizacional (Argote, 2011; Huber, 1991; Lehesvirta, 2004).

Segundo Crossan, Lane e White (1999), a disseminação do conhecimento transcorre pelos níveis (i) individual; (ii) de grupo; e (iii) organizacional. Para percorrer esses níveis, os autores descreveram quatro processos que ocorrem durante a evolução, expansão e disseminação do conhecimento pela estrutura organizacional: (i) intuir; (ii) interpretar; (iii) integrar; e (iv) institucionalizar. A estrutura construída, simbolizando o framework dos 4Is, é apresentada na Figura 1.

\section{Figura 1}

\section{Processo Dinâmico da Aprendizagem Organizacional}

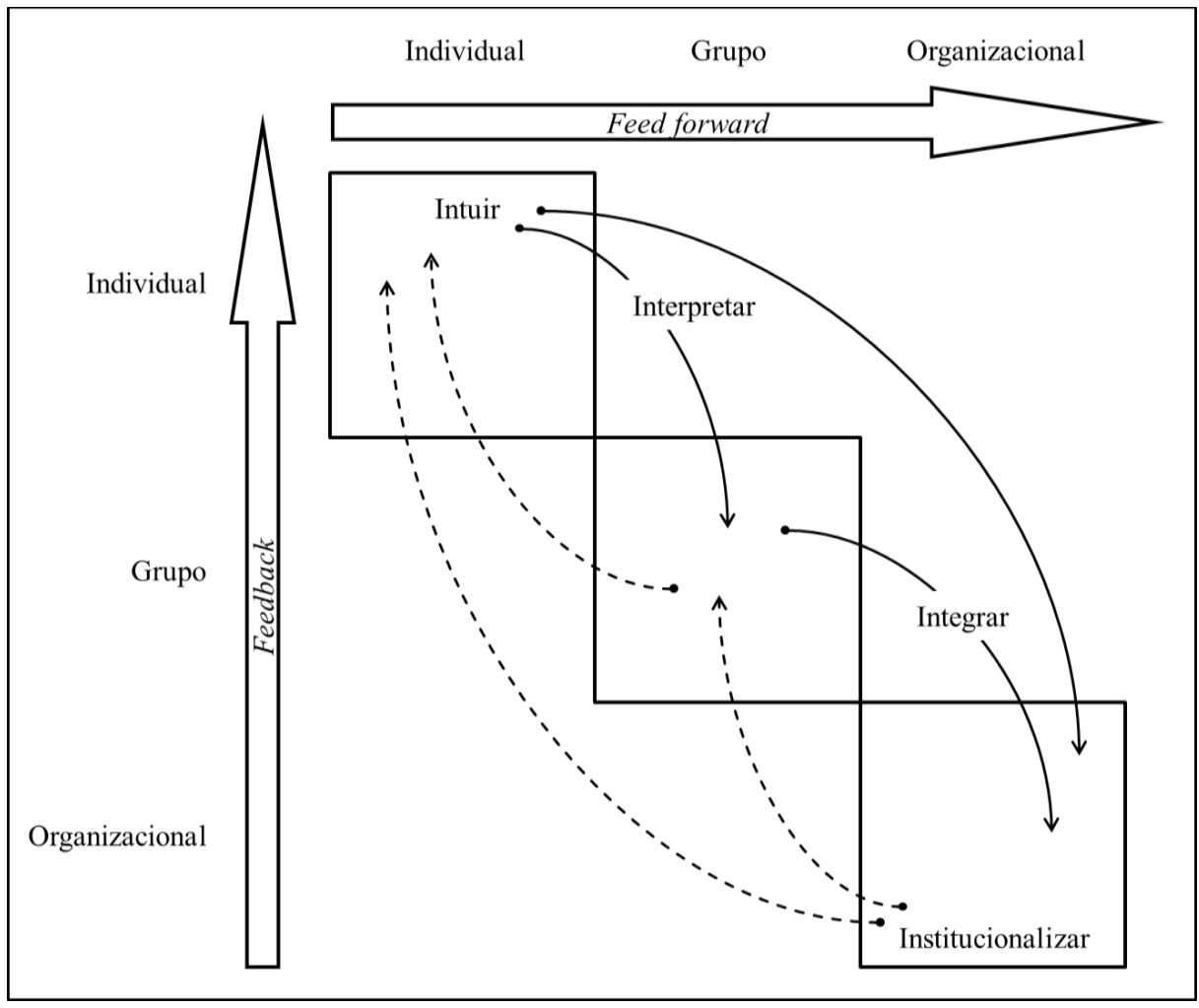

Fonte: Traduzido de Crossan, Lane e White (1999, p. 532).

Intuir diz respeito ao processo iniciado particularmente no indivíduo, relacionado ao conhecimento tácito e pré-consciente (Huang \& Shih, 2011; Mutiganda, 2016; Santos \& Steil, 2015), constituído a partir das experiências pessoais (Goldman et al., 2014), reconhecimento de padrões (Dutta \& Crossan, 2005; Sun \& Anderson, 2010; Waight, 2015), os quais promovem o desenvolvimento de 
novos insights (Bontis et al., 2002; Brettel \& Rottenberger, 2013). Como Kostopoulos, Spanos e Prastacos (2013) afirmam, a intuição está situada dentro dos indivíduos, que exploram, improvisam e desenvolvem soluções, reconhecem oportunidades com base em experiências e conhecimento tácito, do subconsciente.

A partir dos insights, os indivíduos buscam tornar essas informações mais concretas e materializáveis, cristalizando-as junto a outros indivíduos do grupo, buscando uma linguagem comum entre todos (Kostopoulos, Spanos \& Prastacos, 2013; Rikkerink et al., 2016). Esse processo de cristalização é considerado como o de interpretação, e representa a relação entre os indivíduos ao nível de grupo (Bontis et al., 2002; Brettel \& Rottenberger, 2013; Lionzo \& Rossignoli, 2013). Ou seja, definese como uma etapa do processo de explicar, expressar e discutir conscientemente ideias ou insights que, por sua natureza, envolvem mais de um indivíduo (Schlagwein \& Bjorn-Andersen, 2014). A interpretação está diretamente relacionada com a comunicação, com a fala e com ações, nas quais se transmitem informações de um indivíduo para outros (Huang \& Shih, 2011; Schilling \& Kluge, 2009).

O compartilhamento de interpretações individuais, por meio de interações contínuas, tornando um entendimento comum e mais profundo entre as pessoas na organização, promove o processo de integrar o conhecimento (Bontis et al. 2002; Brettel \& Rottenberger, 2013; Schulze et al., 2013). A integração depende e é orientada ao compartilhamento de práticas ou informações para que um entendimento comum e exercícios coletivos sejam adotados (Benn, Edwards \& Argus-Leppan, 2013; Lawrence et al., 2005; Lin \& Sanders, 2017; Santos \& Steil, 2015). A integração permite a ação coletiva, coordenada, coerente e recorrente do grupo, de modo que os membros da equipe concordem e participem em fazê-lo (Ahmad et al., 2015; Jones \& Macpherson, 2006; Kostopoulos, Spanos \& Prastacos, 2013; Lehesvirta, 2004). Assim, o processo de integração realiza a transferência do conhecimento de um grupo para o início de um processo a nível organizacional.

As ações coletivas e o conhecimento construído a partir das constantes interações entre os membros são, então, codificadas e incorporadas à equipe (Kostopoulos, Spanos \& Prastacos, 2013; Zietsma et al., 2002). A incorporação do aprendizado construído a nível individual e em grupo nos elementos não humanos da organização, ou seja, sistemas, estruturas, procedimentos, rotinas e estratégia, de modo que se tornem práticas cotidianas, representam o processo de institucionalizar o conhecimento na organização (Ahmad et al., 2015; Bontis et al., 2002; Crossan \& Berdrow, 2003; Dover \& Lawrence, 2012; Soekijad et al., 2011). Esse processo de incorporação do conhecimento na cultura organizacional que diferencia a ocorrência da institucionalização do conhecimento na organização (Lawrence et al., 2005). A partir da institucionalização, pode-se dizer que ocorreu a aprendizagem organizacional, pois o conhecimento está inserido nas rotinas e repositórios da memória da organização, garantindo sua existência (Crossan, Lane \& White, 1999; Lin \& Sanders, 2017). Do 
contrário, esse conhecimento poderia ser esquecido e se perder durante o tempo devido à falta de codificação e padronização (Kostopoulos, Spanos \& Prastacos, 2013; Lehesvirta, 2004).

Contudo, apesar de definidos e reconhecidos os conceitos sobre os processos que ocorrem para a plena realização da aprendizagem organizacional, estudos apontam que existem lacunas a serem preenchidas em relação ao framework dos 4ls, sobretudo no que diz respeito ao baixo número de pesquisas empíricas, mensurando e avaliando os processos em que ocorrem a disseminação do conhecimento (Berends \& Lammers, 2010; Crossan, Maurer \& White, 2011; Soekijad et al., 2011). Neste sentido, este artigo se posiciona a contribuir para essa lacuna, construindo um arcabouço consolidado para identificar métodos de operacionalização dos processos de aprendizagem organizacional por meio do uso framework dos 4 Is.

\section{Procedimentos metodológicos \\ 3.1 Enquadramento metodológico}

O enquadramento metodológico é compreendido como a análise, a descrição ou a explicação das abordagens e métodos utilizados em uma pesquisa para especificar o conjunto de procedimentos de pressupostos filosóficos ou disciplinas, os quais fundamentam os temas ou finalidades que explicam ou esclarecem o estudo particular para o método científico (Tasca et al., 2010), ou seja, em resumo, apresenta as características científicas do estudo particular para enquadra-lo globalmente.

As características do estudo estão apresentadas segundo quatro elementos: (i) natureza da pesquisa; (ii) abordagem do problema; (iii) fonte de dados; e (iv) procedimentos técnicos.

No que diz respeito à natureza da pesquisa, trata-se de uma pesquisa descritiva, onde se busca evidenciar os procedimentos e métodos encontrados na literatura a respeito da avaliação da aprendizagem organizacional com base no uso do framework proposto por Crossan, Lane e White (1999) e apresentar construtos que permitam a operacionalização de cada um dos processos de aprendizagem organizacional (Gil, 2008).

Tomando-se em consideração que o estudo depende de uma análise reflexiva e interpretação, por parte dos pesquisadores, a respeito dos estudos executados com base no framework dos 4ls, adotase a abordagem qualitativa para analisar os dados coletados (Gray, 2013; Creswell, 2014b). Desta forma, o estudo não busca apenas quantificar os resultados, mas construir um entendimento consolidado para o desenvolvimento dos construtos, conforme se identificam métodos utilizados para avaliação de aprendizagem nas organizações.

O procedimento técnico utilizado para o estudo é a pesquisa bibliográfica (Gray, 2013). Buscando aplicar rigor metodológico ao mapeamento e seleção dos estudos para a análise, procedeuse a uma revisão sistematizada dos estudos que citaram o artigo base de Crossan, Lane e White (1999). Assim, os artigos identificados a partir do processo de seleção se referem aos dados que serão 
analisados para a conclusão do objetivo do trabalho. Deste modo, esse estudo se utiliza de fonte de dados secundária (Gil, 2008).

O resumo do enquadramento metodológico da pesquisa é apresentado na Figura 2.

Figura 2

Resumo do Enquadramento Metodológico da Pesquisa

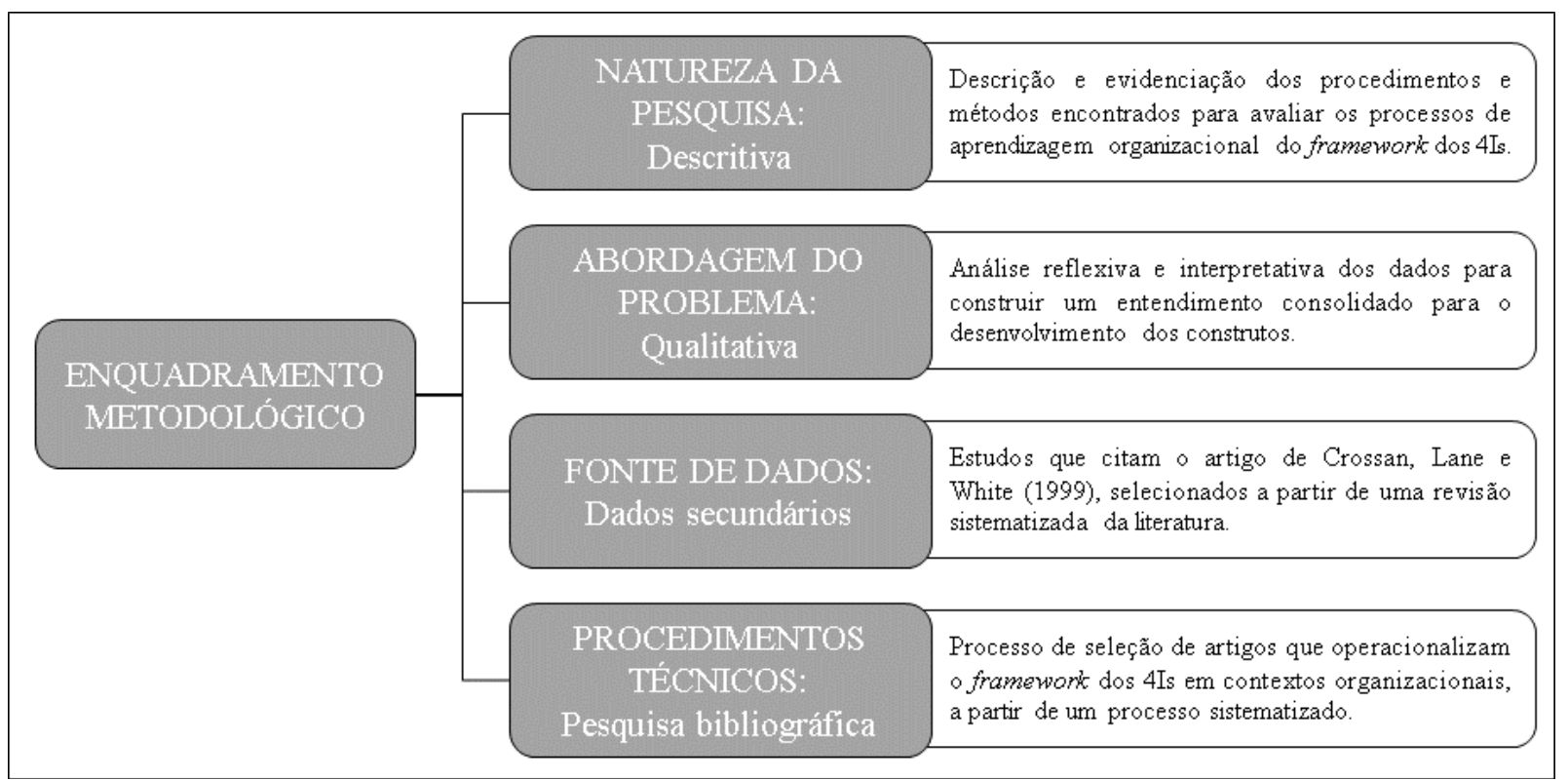

Fonte: Elaborado pelos autores (2018).

\subsection{Processo de seleção e análise dos dados}

O objetivo desta subseção é apresentar o processo executado para selecionar os estudos que fizeram uso do framework dos 4 Is e o operacionalizaram para avaliar a ocorrência dos processos de aprendizagem organizacional. Assim, apresenta-se o processo de revisão sistematizada dos estudos.

A revisão sistematizada, por meio de uma versão simplificada de Ensslin et al. (2015) e Matos et al. (2019), utilizou das bases de dados internacionais Scopus e Web of Science, identificando os trabalhos que referenciaram, se algum modo, o artigo de Crossan, Lane e White (1999), com a intenção de coletar o maior número de trabalhos possível, limitando aos periódicos reconhecidos e indexados pelas referidas bases. Salienta-se, porém, que outras bases de dados foram consideradas para a realização da pesquisa, como EBSCO, Emerald, Engineering Village, ProQuest, Science Direct e Wiley, contudo não possuíam as funcionalidades necessárias para identificação dos trabalhos que referenciaram o artigo base buscado.

Após a coleta dos trabalhos que referenciaram o artigo de Crossan, Lane e White (1999), avaliou-se a aderência das referências frente às delimitações impostas pelos pesquisadores, limitando o volume de trabalhos para análise de conteúdo: (i) artigos publicados em periódicos; (ii) artigos com 
texto integral disponível; (iii) artigos escritos em língua inglesa, portuguesa, francesa ou espanhola; e (iv) artigos que tenham se baseado no framework dos 4ls para evidenciação da aprendizagem. Nesse sentido, são excluídos da base aqueles trabalhos que somente utilizaram o artigo como forma de contextualizar ou para citar aspectos fora do que está estudando.

Como primeira etapa, o acesso ao artigo base, de Crossan, Lane e White (1999), nas duas bases de dados utilizadas, evidenciou a existência de 1.622 trabalhos que o citaram na base Scopus e 998 trabalhos na base Web of Science. O conjunto das referências identificadas, composto por 2.620 referências, é, então, transferido para o gerenciador bibliográfico EndNote (Thomson Reuters, 2013), o qual possibilita a exclusão das referências que não se enquadram nas delimitações impostas, além de permitir a exclusão dos itens repetidos na base de dados. Assim, excluiu-se da base o total de 1.317 referências, duplicadas ou de livros, conferências, entre outros fatores que as enquadravam fora das delimitações impostas pelos pesquisadores. Os demais 1.264 artigos foram acessados um a um, na íntegra, identificando como o framework dos 4Is foi utilizado, diferenciando se o framework foi utilizado para fins de contextualização ou como base do estudo, buscando pelas citações realizadas durante o texto. Deste modo, aqueles artigos que se utilizam de citações de Crossan, Lane e White (1999) apenas para contextualização ou com objetivos diferentes da utilização do framework são excluídos. Por fim, foram selecionados 34 artigos ao final desta etapa.

A partir de uma análise mais reflexiva e detalhada em relação ao uso do framework dos 4ls nos artigos restantes, evidenciou-se que parte do portfólio não operacionalizou o framework estudado, preocupando-se em desenvolver conceitualmente alguns pontos específicos e/ou propondo outras dimensões para composição do framework dos 4ls (Dutta \& Crossan, 2005; Lawrence et al., 2005; Schilling \& Kluge, 2009; Sun \& Anderson, 2010; Dover \& Lawrence, 2012; Guzman, 2015; Lin \& Sanders, 2017). Esses artigos, conceituais em essência, apesar de serem excluídos da base, são utilizados para composição do referencial teórico.

Neste sentido, ao final do processo de seleção dos estudos, concluiu-se um portfólio composto por 27 artigos, que representam os dados para análise específica da operacionalização da avaliação da aprendizagem organizacional por meio do framework dos 4 Is. O resumo do processo de seleção dos materiais é apresentado na Figura 3. 


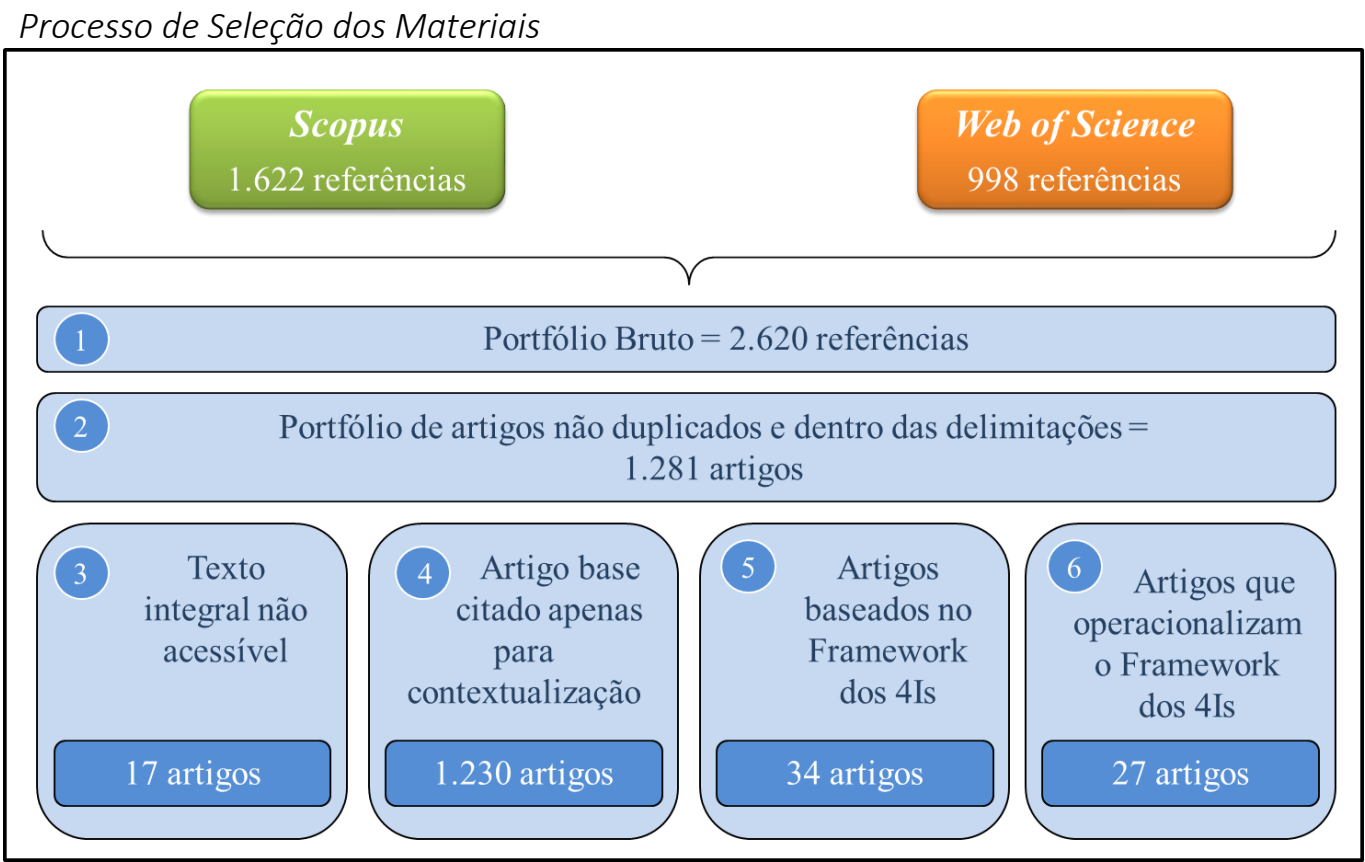

Fonte: Elaborado pelos autores (2018).

O portfólio final de artigos, composto por estudos de operacionalização do framework dos 4Is, fornece informações necessárias para realização da análise e alcance do objetivo do trabalho. Assim, para cada um dos estudos selecionados, foram levantados os procedimentos metodológicos executados nos trabalhos, evidenciando as abordagens, procedimentos técnicos e de coletas de dados. Em um segundo momento, a partir dos relatos identificados nos artigos, relativos a cada um dos processos de aprendizagem organizacional - intuir, interpretar, integrar e institucionalizar - apresentam-se os construtos desenvolvidos para avaliar a operacionalização desses processos, baseados nos estudos selecionados na primeira fase.

Com o fim de aumentar a confiabilidade dos resultados, foi utilizada a estratégia descrita por Creswell (2014a), denominada como member cheking, na qual um dos autores ficou responsável por verificar a coerência dos resultados apontados, executando a interlocução dos achados da pesquisa.

\section{Resultados}

\subsection{Análise dos métodos de pesquisa}

Grande relevância tem sido demonstrada por parte da literatura sobre aprendizagem organizacional para o framework dos 4Is, construído por Crossan, Lane e White (1999), haja vista a quantidade de citações recebida pelo trabalho ao longo dos anos e do reconhecimento de outros estudos (Crossan, Meurer \& White, 2011; Dutta \& Crossan, 2005; Lionzo \& Rossignoli, 2013; Matthews, Maccarthy \& Braziotis, 2017; Mutiganda, 2016). No entanto, foi identificado durante o processo de seleção e análise para composição do portfólio de artigos, que poucos estudos - aproximadamente um 
a cada cem (1\%) - buscaram aprofundar a execução do framework dos 4Is para avaliação da aprendizagem organizacional em contexto empíricos, legitimando com o que Crossan, Mauren e White (2011) já afirmavam sobre as pesquisas que citaram o artigo base do framework dos 4Is.

Desta forma, sobre esse portfólio, composto por 27 artigos, elencam-se os métodos de pesquisa, apresentando as abordagens, procedimentos técnicos executados e de coleta de dados. Esse levantamento é apresentado no Quadro 1, relacionado aos dados identificados nos estudos do portfólio selecionado.

\section{Quadro 1}

Portfólio de Artigos Selecionados e Procedimentos Metodológicos

\begin{tabular}{|c|c|c|c|}
\hline Artigo & $\begin{array}{l}\text { Abordagem de } \\
\text { pesquisa }\end{array}$ & $\begin{array}{l}\text { Procedimentos } \\
\text { Técnicos }\end{array}$ & $\begin{array}{l}\text { Procedimento de } \\
\text { coleta de dados }\end{array}$ \\
\hline $\begin{array}{l}\text { Ahmad, Maynard e } \\
\text { Shanks (2015) }\end{array}$ & $\begin{array}{l}\text { Pesquisa } \\
\text { Qualitativa }\end{array}$ & Estudo de caso & $\begin{array}{l}\text { - Entrevistas } \\
\text { - Análise documental }\end{array}$ \\
\hline $\begin{array}{l}\text { Argyris e Ransbotham } \\
\text { (2016) }\end{array}$ & $\begin{array}{l}\text { Pesquisa } \\
\text { Qualitativa }\end{array}$ & $\begin{array}{l}\text { Estudo de caso } \\
\text { (longitudinal) }\end{array}$ & $\begin{array}{l}\text { - Entrevistas } \\
\text { - Observação participante } \\
\text { - Análise documental }\end{array}$ \\
\hline $\begin{array}{l}\text { Benn, Edwards e Angus- } \\
\text { Leppan (2013) }\end{array}$ & $\begin{array}{l}\text { Pesquisa } \\
\text { Qualitativa }\end{array}$ & Estudo multicaso & - Entrevistas \\
\hline $\begin{array}{l}\text { Berends e Lammers } \\
\text { (2010) }\end{array}$ & $\begin{array}{l}\text { Pesquisa } \\
\text { Qualitativa }\end{array}$ & $\begin{array}{l}\text { Estudo de caso } \\
\text { (longitudinal) }\end{array}$ & $\begin{array}{l}\text { - Observação participante } \\
\text { - Entrevistas } \\
\text { - Pesquisa documental }\end{array}$ \\
\hline $\begin{array}{l}\text { Berson, Da'as e } \\
\text { Waldman (2015) }\end{array}$ & $\begin{array}{c}\text { Pesquisa } \\
\text { Quantitativa }\end{array}$ & Levantamento & - Questionário \\
\hline $\begin{array}{l}\text { Bontis, Crossan e } \\
\text { Hulland (2002) }\end{array}$ & $\begin{array}{c}\text { Pesquisa } \\
\text { Quantitativa }\end{array}$ & Levantamento & $\begin{array}{l}\text { - Questionário } \\
\text { - Entrevistas }\end{array}$ \\
\hline $\begin{array}{l}\text { Brettel e Rottenberger } \\
\text { (2013) }\end{array}$ & $\begin{array}{c}\text { Pesquisa } \\
\text { Quantitativa }\end{array}$ & Levantamento & - Questionário \\
\hline $\begin{array}{l}\text { Cardoso e Caballero } \\
\text { (2013) }\end{array}$ & $\begin{array}{l}\text { Pesquisa } \\
\text { Qualitativa }\end{array}$ & Estudo de caso & $\begin{array}{l}\text { - Entrevistas } \\
\text { - Análise documental } \\
\text { - Observação não-participante }\end{array}$ \\
\hline $\begin{array}{l}\text { Crossan e Berdrow } \\
\text { (2003) }\end{array}$ & $\begin{array}{l}\text { Pesquisa } \\
\text { Qualitativa }\end{array}$ & Estudo de caso & $\begin{array}{l}\text { - Pesquisa documental } \\
\text { - Observação participante } \\
\text { - Entrevistas }\end{array}$ \\
\hline $\begin{array}{l}\text { Dutton, Turner e Lee- } \\
\text { Kelley (2014) }\end{array}$ & $\begin{array}{l}\text { Pesquisa } \\
\text { Qualitativa }\end{array}$ & Estudo de caso & - Entrevistas \\
\hline Goldman et al. (2014) & $\begin{array}{l}\text { Pesquisa } \\
\text { Qualitativa }\end{array}$ & Estudo multicaso & $\begin{array}{l}\text { - Entrevistas } \\
\text { - Observação participante }\end{array}$ \\
\hline Huang e Shih (2011) & $\begin{array}{c}\text { Pesquisa Quali- } \\
\text { Quantitativa }\end{array}$ & Estudo de caso & $\begin{array}{l}\text { - Observação participante } \\
\text { - Entrevistas } \\
\text { - Questionários }\end{array}$ \\
\hline $\begin{array}{l}\text { Jones e Macpherson } \\
\text { (2006) }\end{array}$ & $\begin{array}{l}\text { Pesquisa } \\
\text { Qualitativa }\end{array}$ & Estudos de Caso & $\begin{array}{l}\text { - Entrevistas } \\
\text { - Observação participante } \\
\text { - Análise documental }\end{array}$ \\
\hline \multirow[t]{2}{*}{$\begin{array}{l}\text { Kelliher e Henderson } \\
\text { (2006) }\end{array}$} & $\begin{array}{l}\text { Pesquisa } \\
\text { Qualitativa }\end{array}$ & $\begin{array}{l}\text { Estudo de caso } \\
\text { (longitudinal) }\end{array}$ & $\begin{array}{l}\text { - Observação participante } \\
\text { - Entrevistas } \\
\text { - Análise documental }\end{array}$ \\
\hline & $\begin{array}{c}\text { Pesquisa } \\
\text { Quantitativa }\end{array}$ & Estudos de Caso & $\begin{array}{l}\text { - Entrevistas } \\
\text { - Questionário }\end{array}$ \\
\hline
\end{tabular}




\begin{tabular}{|c|c|c|c|}
\hline Artigo & $\begin{array}{l}\text { Abordagem de } \\
\text { pesquisa }\end{array}$ & $\begin{array}{l}\text { Procedimentos } \\
\text { Técnicos }\end{array}$ & $\begin{array}{l}\text { Procedimento de } \\
\text { coleta de dados }\end{array}$ \\
\hline \multicolumn{4}{|l|}{$\begin{array}{l}\text { Kostopoulos, spanos e } \\
\text { Prastacos (2013) }\end{array}$} \\
\hline Lehervista (2004) & $\begin{array}{l}\text { Pesquisa } \\
\text { Qualitativa }\end{array}$ & Estudo etnográfico & - Observação Participante \\
\hline $\begin{array}{l}\text { Lionzo e Rossignoli } \\
\text { (2013) }\end{array}$ & $\begin{array}{c}\text { Pesquisa } \\
\text { Qualitativa }\end{array}$ & Estudos de Caso & $\begin{array}{l}\text { - Observação participante } \\
\text { - Entrevistas }\end{array}$ \\
\hline $\begin{array}{l}\text { Matthews, MacCarthy e } \\
\text { Brasiotis (2017) }\end{array}$ & $\begin{array}{c}\text { Pesquisa } \\
\text { Qualitativa }\end{array}$ & Estudos de Caso & $\begin{array}{l}\text { - Entrevistas } \\
\text { - Análise documental } \\
\text { - Observação participante }\end{array}$ \\
\hline Mutiganda (2016) & $\begin{array}{l}\text { Pesquisa } \\
\text { Qualitativa }\end{array}$ & Estudo de caso & $\begin{array}{l}\text { - Análise documental } \\
\text { - Entrevistas } \\
\text { - Observação participante }\end{array}$ \\
\hline $\begin{array}{l}\text { Real, Roldán e Leal } \\
\text { (2014) }\end{array}$ & $\begin{array}{c}\text { Pesquisa } \\
\text { Quantitativa }\end{array}$ & Levantamento & - Questionários \\
\hline Rikkerink et al. (2016) & $\begin{array}{c}\text { Pesquisa } \\
\text { Qualitativa }\end{array}$ & $\begin{array}{l}\text { Estudo de caso } \\
\text { (longitudinal) }\end{array}$ & $\begin{array}{l}\text { - Questionários } \\
\text { - Entrevistas } \\
\text { - Análise documental }\end{array}$ \\
\hline Santos e Steil (2015) & $\begin{array}{l}\text { Pesquisa } \\
\text { Qualitativa }\end{array}$ & Estudo de caso & $\begin{array}{l}\text { - Análise documental } \\
\text { - Entrevistas }\end{array}$ \\
\hline $\begin{array}{l}\text { Schlagwein e Bjorn- } \\
\text { Adersen (2014) }\end{array}$ & $\begin{array}{l}\text { Pesquisa } \\
\text { Qualitativa }\end{array}$ & $\begin{array}{l}\text { Estudo de caso } \\
\text { (longitudinal) }\end{array}$ & $\begin{array}{l}\text { - Observação participante } \\
\text { - Entrevistas } \\
\text { - Análise documental }\end{array}$ \\
\hline Schulze et al. (2013) & $\begin{array}{l}\text { Pesquisa } \\
\text { Qualitativa }\end{array}$ & $\begin{array}{l}\text { Estudo de caso } \\
\text { (longitudinal) }\end{array}$ & $\begin{array}{l}\text { - Entrevistas } \\
\text { - Observação participante }\end{array}$ \\
\hline Soekijad et al. (2011) & $\begin{array}{l}\text { Pesquisa } \\
\text { Qualitativa }\end{array}$ & Estudo de caso & $\begin{array}{l}\text { - Entrevistas } \\
\text { - Observação participante } \\
\text { - Análise documental }\end{array}$ \\
\hline Waight (2015) & $\begin{array}{c}\text { Pesquisa } \\
\text { Qualitativa }\end{array}$ & Estudo de campo & - Entrevistas \\
\hline Zietsma et al. (2002) & $\begin{array}{l}\text { Pesquisa } \\
\text { Qualitativa }\end{array}$ & $\begin{array}{l}\text { Estudo de caso } \\
\text { (longitudinal) }\end{array}$ & $\begin{array}{l}\text { - Entrevistas } \\
\text { - Análise documental } \\
\text { - Análise bibliográfica }\end{array}$ \\
\hline
\end{tabular}

Fonte: Elaborado pelos autores (2018).

A visão da abordagem do problema nas pesquisas se refere ao primeiro aspecto levantado (Gray, 2013). O levantamento das abordagens utilizadas pelos artigos demonstra que a grande maioria (78\%) dos estudos relacionados à operacionalização do framework para avaliação da aprendizagem organizacional enxerga o problema de maneira qualitativa, buscando entender e interpretar os fenômenos ocorridos nos contextos específicos para descrevê-los (Creswell, 2014b). Essa abordagem permite compreender como acontecem os processos de aprendizagem e os fluxos de transferência do conhecimento pela estrutura organizacional, preocupando-se com a participação dos indivíduos e dos grupos na construção do conhecimento conjunto.

De outra maneira, os estudos quantitativos preocupam-se com o grau de realização da aprendizagem, a partir de técnicas estatísticas, buscando generalizações quanto ao contexto e ao problema. 
Utilizando-se de uma abordagem mista, o artigo de Huang e Shih (2011) foi o único identificado que abordou a problemática com uma visão quali-quantitativa, aplicando técnicas de observação e análise de entrevistas com a análise estatística para correlacionar aprendizagem com resultados e satisfação dos membros da organização.

Em relação aos procedimentos técnicos identificados nas pesquisas, identificou-se que a maior parte dos trabalhos (70\%) esteve focada em estudar de forma exaustiva um determinado problema em uma organização específica, utilizando-se de estudo de caso (Gil, 2008; Yin, 2010). Dentre esses, em sete artigos os autores descrevem os estudos como longitudinais, onde há a observação e o acompanhamento de situações anteriores e posteriores a situações específicas estudadas relativas às mudanças organizacionais.

Para os estudos de abordagem quantitativa, predomina o procedimento de levantamento, muito por conta nas análises estatísticas, no intuito de correlacionar variáveis de aprendizagem a partir da visão dos processos do framework dos 4ls. Em menor volume, realizaram-se, ainda, estudos multicaso, de campo e etnográfico para análise da aprendizagem nas organizações.

Outra análise de grande relevância para a pesquisa diz respeito ao levantamento dos procedimentos realizados para coleta de dados. Assim, buscou-se identificar os instrumentos e metodologias aplicadas para que os dados pudessem ser resgatados para aplicação das pesquisas. Os resultados evidenciam a importância das pessoas e a forma como visualizam as mudanças cognitivas e de atitudes dos membros da organização, reflexo da ocorrência da aprendizagem. Para identificar essas percepções, o procedimento mais utilizado foi a execução de entrevistas com os indivíduos das organizações, sejam gestores, líderes de equipes e outros membros ou stakeholders, presente em 85\% dos estudos analisados. A relevância dada às entrevistas diz respeito à identificação das percepções, sensibilidade e identificação dos pontos de vista inerentes a atividade da organização pelas pessoas que vivenciaram o seu cotidiano e suas rotinas, bem como a visão dos processos que levaram a mudanças nas instituições. Desta forma, é possível depreender que as entrevistas são os principais instrumentos para avaliação dos processos de aprendizagem que ocorrem na estrutura organizacional. Pode-se citar, como exemplo, que a partir das entrevistas os estudos puderam perceber como ocorreu a estruturação dos insights (processo de intuir), originários nos indivíduos, em conhecimentos compartilhados com os grupos para sua efetiva execução, junto à ação coerente por parte dos membros dos grupos (processo de interpretar e integrar), e efetiva sistematização na organização (processo de institucionalizar), evidenciando relevantes informações aos investigadores (Crossan \& Berdrow, 2003; Santos \& Steil; 2015; Schlagwein \& Bjorn-Adersen, 2014; Taylor, Bogdan \& Devault, 2015).

Ainda, apresentam representatividade no portfólio selecionado os procedimentos de observação participante e análise documental, presentes em quase metade dos artigos analisados. A observação diz respeito à inserção do investigador no ambiente organizacional, buscando identificar o 
comportamento e as atividades dos indivíduos da organização, descrevendo e reportando as situações anteriores e posteriores a mudanças, e os comportamentos e ações percebidas em relação aos indivíduos e aos grupos (Argyris \& Ransbotham, 2016; Berends \& Lammers, 2010; Huang \& Shih, 2011). Para isso, os pesquisadores se comunicam com as pessoas de maneira mais informal, para compreender os processos, modo de agir das pessoas e verificar como os objetivos estratégicos da organização se modificam frente à aprendizagem materializada nos elementos não-humanos da organização. Reforçase, deste modo, que o referido procedimento apresenta grande relevância para a abordagem qualitativa do problema de pesquisa, visto que os pesquisadores se inserem no cotidiano da organização para perceber alterações nas práticas, comportamentos e atitudes das pessoas na empresa, permitindo uma análise mais confiável e fiel à realidade (Goldman et al., 2014; Lionzo \& Rossignoli, 2013; Taylor, Bogdan \& Devault, 2015).

Semelhantemente, a coleta de documentos para realização da análise também foi utilizada pelos trabalhos. No entanto, esses dados são mais utilizados como fonte adicional de informações, fornecendo mais robustez aos resultados. Documentos internos das organizações, como relatórios e memorandos internos, sistemas gráficos, atas de reuniões, manuais, apostilas, regimentos, tutoriais em vídeo, apresentações, bancos de dados, entre outros, foram analisados para identificar mudanças nos elementos não-humanos da organização, como procedimentos, sistemas, estruturas e demais fatores que possam demonstrar como a aprendizagem afetou a organização durante o período estudado (Jones \& Bacpherson, 2006; Santos \& Steil, 2015; Soekijad et al., 2011).

Assim, conclui-se que a fonte de dados primária (Grey, 2013) possui papel fundamental para a percepção da ocorrência da aprendizagem organizacional, a partir da observação e compreensão dos processos que fluem pela estrutura da organização para transferir o conhecimento, vivenciando e percebendo a realidade dos contextos organizacionais.

\subsection{Construtos para operacionalização dos processos do framework dos $41 \mathrm{~s}$}

Os estudos identificados e selecionados para o portfólio representam importante arcabouço para compreender os métodos de operacionalização dos processos definidos no framework dos $4 \mathrm{Is}$. A partir dos relatos encontrados em cada um dos estudos, pode-se construir um construto composto pela consolidação de métodos e formas utilizadas para avaliação dos processos nas organizações.

Um construto deve apresentar a relação de um conceito com o mundo real, baseado em fenômenos observáveis e mensuráveis para operacionaliza-lo, ou seja, consiste na transformação de conceitos teóricos em variáveis observáveis para serem mensuradas ou avaliadas por meio de instrumentos apropriados. Assim, o construto possui um significado construído intencionalmente a partir de um fundamento teórico e possui a intenção de especificar claramente suas características, de modo permitir sua observação e mensuração (Martins, 2005). 
Neste sentido, com base no empirismo evidenciado nos estudos, procedeu-se ao desenvolvimento dos construtos para os processos relacionados à Aprendizagem Organizacional, baseados no framework dos 4ls (Crossan, Lane \& White, 1999).

O Quadro 2 evidencia o construto desenvolvido para o processo de Intuir, composto pelos autores que, de alguma maneira, abordaram a operacionalização deste processo. Conforme os elementos identificados, faz-se necessária uma reflexão quanto aos aspectos inerentes ao indivíduo, buscando compreender como se desenvolvem e se manifestam os conhecimentos.

\section{Quadro 2}

\section{Construto Para oProcesso Intuir}

\begin{tabular}{|c|c|}
\hline Construto & Intuir \\
\hline Definição & $\begin{array}{l}\text { Intuir é o reconhecimento pré-consciente de padrões e/ou possibilidades inerentes em um } \\
\text { fluxo de experiências pessoais. Esse processo pode afetar o comportamento intuitivo de um } \\
\text { indivíduo, mas só afeta a outros quando há a tentativa de interagir com esse indivíduo. }\end{array}$ \\
\hline Operacionalização & $\begin{array}{l}\text { - Os indivíduos possuem conhecimento sobre os objetivos estratégicos da organização; } \\
\text { - Os indivíduos são capazes de enxergar problemas e situações de novas e diferentes } \\
\text { maneiras; } \\
\text { - Os indivíduos costumam gerar novos insights; } \\
\text { - Os indivíduos demonstram utilizar de experiências passadas para construir novas ideias; } \\
\text { - Os indivíduos demonstram iniciativa para propor novas perspectivas à organização; } \\
\text { - Indivíduos se utilizam de metáforas para explanar ideias; } \\
\text { - Indivíduos questionam processos formalizados na estrutura organizacional; }\end{array}$ \\
\hline Fonte & $\begin{array}{l}\text { Crossan, Lane e White (1999); Bontis, Crossan e Hulland (2002); Brettel e Rottenberger } \\
\text { (2013); Cardoso e Caballero (2013); Crossan e Berdrow (2003); Kostopoulos, Spanos e } \\
\text { Prastacos (2013); Lawrence et al. (2005); Lin e Sanders (2017); Mutiganda (2016); Santos e } \\
\text { Steil (2015); Schlagwein e Bjorn-Andersen (2014); Waight (2015); Zietsma et al. (2002). }\end{array}$ \\
\hline
\end{tabular}

Fonte: Elaborado pelo autor com base em diversos autores.

Quanto ao processo Interpretar, percebe-se que a operacionalização, abordada nos diferentes trabalhos, é tratada como um momento de expansão do conhecimento tácito do indivíduo, a partir do compartilhamento de ideias, discussão e experimentação com outros indivíduos do grupo. O construto desenvolvido para o processo Interpretar está apresentado no Quadro 3. 


\section{Quadro 3}

\section{Construto Para o Processo Interpretar}

\begin{tabular}{|l|l|}
\hline Construto & Interpretar \\
\hline Definição & Interpretar é a explicação de uma visão ou uma ideia a si ou para os outros. Esse processo \\
& passa do pré-verbal ao verbal e requer o desenvolvimento de uma linguagem comum. \\
\hline Operacionalização & - Durante as reuniões são discutidas novas ideias; \\
& - As pessoas são encorajadas a proporem novas ideias e novos pontos de vista; \\
& - Os indivíduos costumam expor seus pontos de vista com o grupo; \\
& - Novas perspectivas são discutidas entre o grupo; \\
& - O(s) gestor(es) incentiva(m) o comportamento pró-ativo dos indivíduos; \\
& - Percebe-se experimentação por parte dos indivíduos do grupo em relação a novas ideias; \\
& - Membros do grupo interpretam e expandem as ideias conjuntamente; \\
& - Percebe-se o desenvolvimento de mapas cognitivos para expandir conhecimento sobre \\
& novas ideias; \\
& $\begin{array}{l}\text { Crossan, Lane e White (1999); Ahmad, Maynard e Shanks (2015); Bontis, Crossan e Hulland } \\
\text { (2002); Brettel e Rottenberger (2013); Cardoso e Caballero (2013); Crossan e Berdrow } \\
\text { (2003); Dover e Lawrence (2012); Dutta e Crossan (2005); Huang e Shih (2011); } \\
\text { Konte }\end{array}$ \\
\hline Kostopoulos, Spanos e Prastacos (2013); Lin e Sanders (2017); Lionzo e Rossignoli (2013); \\
Andersen (2014); Schulze et al. (2013); Zietsma et al. (2002). \\
\hline
\end{tabular}

Fonte: Elaborado pelo autor com base em diversos autores.

A operacionalização do processo de Integrar foi identificada, com base nos artigos analisados, como ações coerentes e coletivas entre os membros do grupo. A partir da ação intencional e o conhecimento mútuo entre os participantes do grupo, reconhece-se que há integração entre o conhecimento dos indivíduos. O construto desenvolvido para o processo Integrar está apresentado no Quadro 4. 


\section{Quadro 4}

Construto oara o Processo Integrar

\begin{tabular}{|l|l|}
\hline Construto & Integrar \\
\hline Definição & Integrar é o processo de desenvolvimento de entendimento compartilhado entre os \\
& indivíduos e a tomada de ação coordenada por meio de ajustes mútuos. Diálogo e ação \\
& conjunta são essenciais para o desenvolvimento do entendimento compartilhado. Esse \\
& processo inicia de maneira pontual e informal, mas se as ações coordenadas são exercidas \\
& o conhecimento é institucionalizado. \\
\hline Operacionalização & - Percebe-se ação conjunta e coletiva no grupo; \\
& - Indivíduos compartilham do conhecimento com outros membros do grupo; \\
& - Busca-se o entendimento comum entre todos os indivíduos do grupo; \\
- Programas de treinamento e/ou capacitação são executados para que todos os & indivíduos compartilhem do conhecimento; \\
& - Percebem-se ações coerentes por parte dos indivíduos da organização, em relação a \\
novas perspectivas delineadas; & Crossan, Lane e White (1999); Argyris e Ransbotham (2016); Benn, Edwards e Angus- \\
Leppan (2013); Bontis, Crossan e Hulland (2002); Brettel e Rottenberger (2013); Crossan e \\
Berdrow (2003); Dutta e Crossan (2005); Huang e Shih (2011); Rikkerink et al. (2016); \\
Santos e Steil (2015); Schlagwein e Bjorn-Andersen (2014); Soekijad et al. (2011); Waight \\
(2015).
\end{tabular}

Fonte: Elaborado pelo autor com base em diversos autores.

Por fim, o construto para o processo Institucionalizar faz referência aos elementos nãohumanos da organização. Os artigos que abordam o processo lidam com as rotinas, normatização, estruturas, manuais, dentre outros artefatos, onde se asseguram que o conhecimento esteja estabelecido e seja acessível a todos os indivíduos da organização, para que haja ações congruentes aos objetivos estratégicos definidos. O construto para o processo Institucionalizar está evidenciado no Quadro 5. 


\section{Quadro 5}

\section{Construto para o Processo Institucionalizar}

\begin{tabular}{|c|c|}
\hline Construto & Institucionalizar \\
\hline Definição & $\begin{array}{l}\text { Institucionalizar é o processo de assegurar a ocorrência de ações rotineiras. As tarefas são } \\
\text { definidas, ações especificadas e mecanismos organizacionais implementados para garantir } \\
\text { que determinadas ações aconteçam. Institucionalizar é o processo de incorporação da } \\
\text { aprendizagem que tem sido construída nos indivíduos e grupos nas instituições da } \\
\text { organização, incluindo sistemas, estruturas, procedimentos e estratégias. }\end{array}$ \\
\hline Operacionalização & $\begin{array}{l}\text { - Os novos procedimentos estão formalizados nas estruturas não-humanas da } \\
\text { organização; } \\
\text { - Incorporação do aprendizado individual e de grupo nos elementos não-humanos da } \\
\text { organização; } \\
\text { - Recomendações dos grupos ou indivíduos são incorporadas pela organização; } \\
\text { - Os objetivos estratégicos da organização se modificaram; } \\
\text { - Estruturas, procedimentos, manuais, sistemas, entre outros, são alterados para refletir a } \\
\text { nova estratégia organizacional; } \\
\text { - Novas diretrizes são formuladas e comunicadas aos membros da organização; } \\
\text { - Percebe-se que os membros da organização conhecem os novos objetivos estratégicos; } \\
\text { - Avaliação das novas ações rotinizadas; }\end{array}$ \\
\hline Fonte & $\begin{array}{l}\text { Crossan, Lane e White (1999); Ahmad, Maynard e Shanks (2015); Bontis, Crossan e Hulland } \\
\text { (2002); Brettel e Rottenberger (2013); Cardoso e Caballero (2013); Crossan e Berdrow } \\
\text { (2003); Dutta e Crossan (2005); Huang e Shih (2011); Jones e Macpherson (2006); } \\
\text { Kostopoulos, Spanos e Prastacos (2013); Lin e Sanders (2017); Mutiganda (2016); Santos e } \\
\text { Steil (2015); Schlagwein e Bjorn-Andersen (2014); Schulze et al. (2013); Waight (2015); } \\
\text { Zietsma (2002). }\end{array}$ \\
\hline
\end{tabular}

Fonte: Elaborado pelo autor com base em diversos autores.

O conjunto de artigos analisados permitiu identificar fatores para mensurar a ocorrência da aprendizagem organizacional, os quais são consolidados nos construtos desenvolvidos. Espera-se que, assim, forneça-se uma contribuição para os estudos referentes à aprendizagem organizacional, principalmente no que se refere ao framework dos 4 Is.

\section{Considerações finais}

Em virtude das grandes e mutáveis demandas que atingem as organizações, levando à necessidade de melhorias constantes e adaptabilidade no mercado, tem-se buscado por processos de gestão que auxiliem e apoiem as pessoas na estrutura organizacional para tomar os rumos corretos dos 
negócios. Nesse sentido, o tema de aprendizagem organizacional tem recebido especial atenção na literatura sobre gestão. Em especial, o framework dos 4Is (Crossan, Lane \& White, 1999) ganhou relevância no tema, apresentando os processos pelos quais ocorrem a aprendizagem nas organizações, desde o conhecimento individual, com o processo de intuir, evoluindo para o conhecimento em grupo com os processo de interpretar e integrar, efetivando a aprendizagem na organização por meio do processo de institucionalizar.

No entanto, ainda que relevante e reconhecido cientificamente (Benn, Edwards \& AngusLeppan, 2013; Berson, Da'as \& Waldman, 2015), não fica claro no estudo de Crossan, Lane e White (1999) e nos artigos seguintes como se avaliar o processo de AO em seus processos de aprendizagem. Assim, esse trabalho se propõe a consolidar, com base nos estudos que fizeram uso do framework, o entendimento a respeito dos referidos processos, reunindo métodos e procedimentos utilizados para avaliar a ocorrência da aprendizagem nas organizações. Para alcançar o resultado proposto, esse trabalho teve por objetivo desenvolver construtos para operacionalização dos processos de aprendizagem organizacional e permitir sua avaliação.

Buscando atender ao objetivo, primeiramente desenvolveu-se uma revisão sistematizada, para construir um robusto portfólio de artigos identificados a partir do uso do framework estudado. Por meio da identificação dos artigos que citaram o trabalho de Crossan, Lane e White (1999), utilizando-se das bases de dados internacionais Scopus e Web of Science, realizou-se a composição de um banco de artigos. Com o apoio de um software gerenciador bibliográfico, foram acessados, um a um, integralmente, os textos dos artigos. Aqueles artigos que citaram o artigo de Crossan, Lane e White (1999) somente para fins de contextualização foram excluídos, restando para análise 27 artigos que operacionalizaram o framework dos $4 \mathrm{Is}$, os quais compuseram o portfólio de artigos para análise dos métodos e procedimentos de avaliação da aprendizagem organizacional.

Os resultados da análise foram divididos em dois momentos, compostos pelos procedimentos metodológicos primeiramente e, no segundo momento, com o desenvolvimento dos construtos para mensuração, operacionalização e avaliação dos processos de AO.

Em relação aos procedimentos metodológicos, evidenciou-se a ênfase para os estudos de caso, com abordagem qualitativa, instrumentalizados a partir de entrevistas, observação participante e análise documental, as quais representam os métodos mais aplicados dentre aqueles do portfólio. Demonstra-se, assim, a importância da vivência e da interação direta, por parte do investigador, com a rotina e o cotidiano das organizações para identificar como ocorre a aprendizagem e como se dão os processos.

Finalmente, apresentaram-se os construtos desenvolvidos com base nos estudos analisados. Com a definição dos construtos, busca-se contribuir para o tema com um arcabouço conceitual, construído a partir do empirismo identificado e relatado em estudos já publicados sobre o tema, no 
qual se apresentam métodos e formas executadas para identificação da ocorrência da aprendizagem. Assim, o artigo fornece orientações a respeito das formas de operacionalização da avaliação da aprendizagem organizacional, agregando conhecimento à área de gestão e da aprendizagem organizacional.

\section{Referências}

Ahmad, A., Maynard, S. B., \& Shanks, G. (2015). A case analysis of information systems and security incident responses. International Journal of Information Management, 35(6), 717-723.

Argote, L. (2011). Organizational learning research: Past, present and future. Management learning, 42(4), 439-446.

Argyris, Y., \& Ransbotham, S. (2016). Knowledge entrepreneurship: institutionalising wiki-based knowledge-management processes in competitive and hierarchical organisations. Journal of Information Technology, 31(2), 226-239.

Benn, S., Edwards, M., \& Angus-Leppan, T. (2013). Organizational learning and the sustainability community of practice: The role of boundary objects. Organization \& Environment, 26(2), 184202.

Berends, H., \& Lammers, I. (2010). Explaining discontinuity in organizational learning: A process analysis. Organization Studies, 31(8), 1045-1068.

Berson, Y., Da'as, R. a., \& Waldman, D. A. (2015). How do leaders and their teams bring about organizational learning and outcomes? Personnel Psychology, 68(1), 79-108.

Bontis, N., Crossan, M. M., \& Hulland, J. (2002). Managing an organizational learning system by aligning stocks and flows. Journal of management studies, 39(4), 437-469.

Brettel, M., \& Rottenberger, J. D. (2013). Examining the link between entrepreneurial orientation and learning processes in small and medium-sized enterprises. Journal of Small Business Management, 51(4), 471-490.

Cardoso, O., \& Caballero, N. (2013). A co-gestão e a aprendizagem organizacional no modelo 4l: O caso esporte clube Juventude. Espacios, 34(2).

Creswell, J. W. (2014a). A concise introduction to mixed methods research: Sage Publications.

Creswell, J. W. (2014b). Investigação Qualitativa e Projeto de Pesquisa-: Escolhendo entre Cinco Abordagens: Penso Editora.

Crossan, M. M., \& Berdrow, I. (2003). Organizational learning and strategic renewal. Strategic management journal, 24(11), 1087-1105.

Crossan, M. M., Lane, H. W., \& White, R. E. (1999). An organizational learning framework: From intuition to institution. Academy of management review, 24(3), 522-537.

Crossan, M. M., Maurer, C. C., \& White, R. E. (2011). Reflections on the 2009 AMR decade award: do we have a theory of organizational learning? Academy of management review, 36(3), 446-460. 
Dover, G., \& Lawrence, T. B. (2012). The role of power in nonprofit innovation. Nonprofit and Voluntary Sector Quarterly, 41(6), 991-1013.

Dutta, D. K., \& Crossan, M. M. (2005). The nature of entrepreneurial opportunities: Understanding the process using the 41 organizational learning framework. Entrepreneurship Theory and Practice, 29(4), 425-449.

Dutton, C., Turner, N., \& Lee-Kelley, L. (2014). Learning in a programme context: An exploratory investigation of drivers and constraints. International Journal of Project Management, 32(5), 747-758.

Easterby-Smith, M., Crossan, M., \& Nicolini, D. (2000). Organizational learning: debates past, present and future. Journal of management studies, 37(6), 783-796.

Ensslin, S. R., Ensslin, L., Matos, L. d. S., Dutra, A., \& Ripoll-Feliu, V. M. (2015). Research opportunities in performance measurement in public utilities regulation. International Journal of Productivity and Performance Management, 64(7), 994-1017.

Gil, A. C. (2008). Métodos e técnicas de pesquisa social: 6. ed. Ediitora Atlas SA.

Goldman, E., Wesner, M., M. Plack, M., N. Manikoth, N., \& Haywood, Y. (2014). Secondhand learning from graduates of leadership development programs. Journal of Workplace Learning, 26(8), 511-528.

Gray, D. E. (2013). Doing research in the real world: Sage.

Guzmán, J. A. A. (2015). El aprendizaje organizacional desde un enfoque cognitivo. 3c Empresa: investigación y pensamiento crítico, 4(2), 136-148.

Huang, Y., \& Shih, H.-C. (2011). A new mode of learning organization. International Journal of Manpower, 32(5/6), 623-644.

Huber, G. P. (1991). Organizational learning: The contributing processes and the literatures. Organization science, 2(1), 88-115.

Jones, O., \& Macpherson, A. (2006). Inter-organizational learning and strategic renewal in SMEs: Extending the 4 I framework. Long Range Planning, 39(2), 155-175.

Kelliher, F., \& Bernadette Henderson, J. (2006). A learning framework for the small business environment. Journal of European Industrial Training, 30(7), 512-528.

Kostopoulos, K. C., Spanos, Y. E., \& Prastacos, G. P. (2013). Structure and function of team learning emergence: A multilevel empirical validation. Journal of Management, 39(6), 1430-1461.

Lawrence, T. B., Mauws, M. K., Dyck, B., \& Kleysen, R. F. (2005). The politics of organizational learning: integrating power into the 4 I framework. Academy of management review, 30(1), 180-191.

Lehesvirta, T. (2004). Learning processes in a work organization: From individual to collective and/orvice versa? Journal of Workplace Learning, 16(1/2), 92-100.

Lin, C. H., \& Sanders, K. (2017). HRM and innovation: a multi-level organisational learning perspective. Human Resource Management Journal, 27(2), 300-317. 
Lionzo, A., \& Rossignoli, F. (2013). Knowledge integration in family SMEs: an extension of the 41 model. Journal of Management \& Governance, 17(3), 583-608.

March, J. G. (1991). Exploration and exploitation in organizational learning. Organization science, 2(1), 71-87.

Matos, L. S., Valmorbida, S. M. I., Martins, V. A., \& Ensslin, S. R. (2019). Development of performance evaluation theme: a systematic analysis of the literature. Contextus - Revista Contemporânea de Economia e Gestão, 17 (2), 63-97.

Martins, G. d. A. (2005). Sobre conceitos, definições e constructos nas ciências administrativas. Gestão \& Regionalidade, 22(62).

Matthews, R. L., MacCarthy, B. L., \& Braziotis, C. (2017). Organisational learning in SMEs: a process improvement perspective. International Journal of Operations \& Production Management, 37(7), 970-1006.

Mutiganda, J. C. (2016). Financial management of public services in a hybrid organisation: a learning approach in inter-organisational settings. International Journal of Public Sector Performance Management, 2(4), 310-330.

Real, J. C., Roldán, J. L., \& Leal, A. (2014). From entrepreneurial orientation and learning orientation to business performance: analysing the mediating role of organizational learning and the moderating effects of organizational size. British Journal of Management, 25(2), 186-208.

Rikkerink, M., Verbeeten, H., Simons, R.-J., \& Ritzen, H. (2016). A new model of educational innovation: Exploring the nexus of organizational learning, distributed leadership, and digital technologies. Journal of Educational Change, 17(2), 223-249.

Santos, J. L. S., \& Steil, A. V. (2015). Organizational learning and power dynamics: a study in a Brazilian University. The Learning Organization, 22(2), 115-130.

Schilling, J., \& Kluge, A. (2009). Barriers to organizational learning: An integration of theory and research. International journal of management reviews, 11(3), 337-360.

Schlagwein, D., \& Bjørn-Andersen, N. (2014). Organizational learning with crowdsourcing: The revelatory case of LEGO. Journal of the Association for Information Systems, 15(11), 754-778.

Schulze, A., Schmitt, P., Heinzen, M., Mayrl, P., Heller, D., \& Boutellier, R. (2013). Exploring the 41 framework of organisational learning in product development: value stream mapping as a facilitator. International Journal of Computer Integrated Manufacturing, 26(12), 1136-1150.

Soekijad, M., van den Hooff, B., Agterberg, M., \& Huysman, M. (2011). Leading to learn in networks of practice: two leadership strategies. Organization Studies, 32(8), 1005-1027.

Sun, P. Y., \& Anderson, M. H. (2010). An examination of the relationship between absorptive capacity and organizational learning, and a proposed integration. International journal of management reviews, 12(2), 130-150.

Tasca, J. E., Ensslin, L., Ensslin, S. R., \& Alves, M. B. M. (2010). An approach for selecting a theoretical framework for the evaluation of training programs. Journal of European Industrial Training, 34(7), 631-655. 
Taylor, S. J., Bogdan, R., \& DeVault, M. (2015). Introduction to qualitative research methods: A guidebook and resource. John Wiley \& Sons.

Thomson Reuters. (2013). EndNote X.7.ed.: Thomson Reuters.

Waight, C. L. (2015). Learning During the Integration Phase of Mergers and Acquisitions: Perspectives From Learning and Development Professionals. Performance Improvement Quarterly, 28(1), 726.

Yin, R. K. (2010). Estudo de Caso: Planejamento e Métodos (Vol. 4. ed.): Bookman editora.

Zietsma, C., Winn, M., Branzei, O., \& Vertinsky, I. (2002). The war of the woods: Facilitators and impediments of organizational learning processes. British Journal of Management, 13(S2), S61-S74. 